\title{
Whites Science
}

A Scientific Erudition

\section{MALIGNANT PERIPHERAL NERVE SHEATH TUMOR OF INDEX FINGER: A RARE PRESENTATION}

\author{
Rana K Sherwani ${ }^{1}$, Kafil Akhtar, Murad Ahmad, ${ }^{*}$ Mazhar Abbas \\ The Department of Pathology and *Orthopaedic Surgery, Jawaharlal Nehru Medical College, Aligarh Muslim \\ University, Aligarh, India
}

\begin{abstract}
Malignant peripheral nerve sheath tumor is a rare sarcoma with ecto-mesenchymal origin. Intraosseous origin of MPNST is very rare and it can be associated with neurofibromatosis type 1 with an incidence of 5-42\% in such patients. MPNSTs have rarely been reported in unusual body parts like head, neck and bone. Here we report a rare occurrence of MPNST in the right index finger where the tumor was found to arise from the soft tissue of index finger, involving the underlying bone.
\end{abstract}

Key Words: Bone, Index finger, Immunohistochemistry, MPNST.

\section{INTRODUCTION}

Sarcoma arising from a peripheral nerve is known as malignant peripheral nerve sheath tumor (MPNST). It was previously known as neurofibrosarcoma, neurogenic sarcoma, malignant neurilemmoma, and malignant schwannoma. MPNST comprise $5 \%$ of all soft tissue sarcomas, with an incidence of $0.001 \%$ in the general population. It can be associated with neurofibromatosis type 1 with an incidence of 5$42 \%$ in such patients. ${ }^{1}$

The anatomical sites commonly affected are proximal portions of the upper and lower extremities and the trunk. MPNSTs have rarely been reported in other unusual body parts like head, neck and bone. ${ }^{2}$ These tumors often create diagnostic problems because of their cellular origin and histopathological similarities with other spindle cell sarcomas like monophasic synovial sarcoma, leiomyosarcoma and fibrosarcoma. Thus, a combination of gross, histopathological and immunohistochemical studies are used for diagnosing these tumors. Here we report a rare occurrence of MPNST in the right index finger with involvement of the underlying bone.

\section{CASE SUMMARY}

*Corresponding author:

Email id: drkafilakhtar@gmail.com http://dx.doi.org/10.20530/IJIMHS 8 24-26 ISSN 2056-9866 @ 2016
A 35year old male presented with a diffuse mass of $6 \times 4 \times 3 \mathrm{~cm}$ in the right index finger, associated with pain and loss of sensation since a month. On palpation, the swelling was tender, firm in consistency and fixed to the underlying tissue. On general examination, the patient was of average built and nutrition with no pallor, icterus, cyanosis, clubbing, pedal edema, lymphadenopathy or similar swelling in any other part of the body.

Fine needle aspiration biopsy showed plump to spindle cells with fine granular chromatin and distinct nucleoli and ill-defined cell outline, suggestive of a malignant spindle cell neoplasm. (Figure 1)

Fig.1. Fine needle aspiration biopsy showed plump to spindle cells with fine granular chromatin and distinct nucleoli and ill-defined cell outline, suggestive of a malignant spindle cell neoplasm.

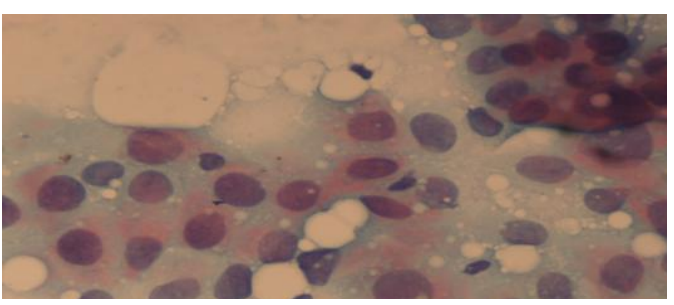

The index finger was amputated which showed an irregular yellow white firm mass with foci of haemorrhage and necrosis. (Figure 2) 
Fig.2. The index finger showed an irregular yellow white firm mass with foci of haemorrhage and necrosis.

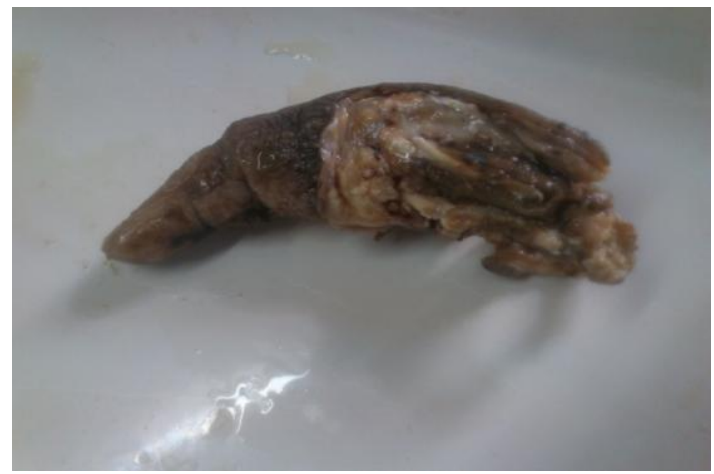

Hematoxylin and eosin stained tissue sections showed interlacing bundles of nerve fibers in a wavy pattern with marked cytological atypia. (Figure 3)

Fig.3. Tissue sections showed interlacing bundles of nerve fibers in a wavy pattern with marked cytological atypia. Hematoxylin and Eosin $x 40 x$

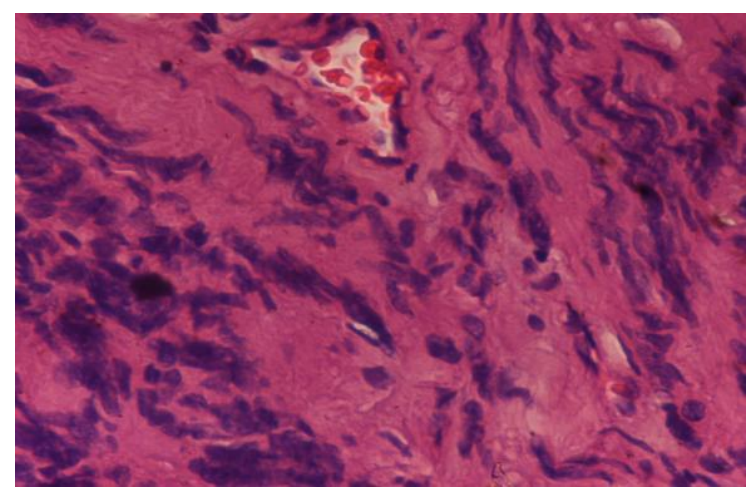

Fig.4. Immunohistochemistry showed diffuse cytoplasmic positivity of S-100 protein in the tumor cells. IHC x S-100

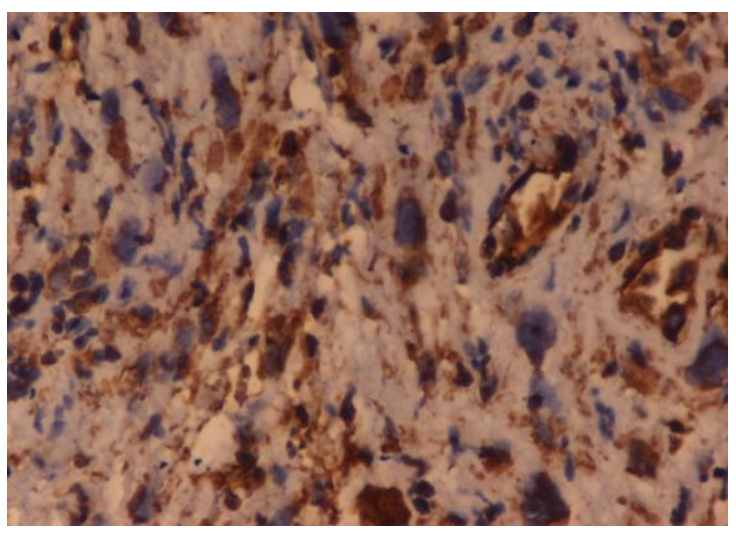

cytoplasmic positivity of S100 protein in the tumor cells. Figure 4)
On follow up, our patient is doing well, without any evidence of recurrence.

\section{DISCUSSION}

Malignant peripheral nerve sheath tumor is a rare sarcoma with ecto-mesenchymal origin. Sites commonly involved are deeper soft tissues usually in the proximal portion of a nerve trunk and the sciatic nerve, brachial plexus and the sacral plexus are most often affected. ${ }^{3}$ MPNSTs involving other body parts are extremely rare, though cases have been reported arising from cranial nerve, facial nerve and the orbit. ${ }^{1,4}$ Intraosseous origin of MPNST in the spine is very rare, with an unclear pathogenesis, though it has been suggested to arise by the proliferation of entrapped neural crest cells during the development of the vertebral body. ${ }^{5} \quad$ Patnaik et al ${ }^{3}$ reported a primary intraosseous MPNST of the spine with a giant paraspinal and retrospinal subcutaneous extension. Iwama et $\mathrm{al}^{4}$ have reported a case of MPNST of the distal phalanx of the fifth toe. In our case, the tumor was found to arise from the soft tissue of index finger, involving the underlying bone.

MPNST is associated with neurofibromatosis in 5 to $42 \%$ cases and the estimated the risk of MPNST in neurofibromatosis to be 4600 times greater than the general population. ${ }^{6}$ Most of the patients present in the second and third decades of life, but when NF1 is present, MPNST tends to occur earlier. ${ }^{7}$ Clinically most cases present with a large fleshy, often necrotic mass with a size of more than $5 \mathrm{~cm}$ with pain and symptoms of nerve defects. The tumor in our case was white, firm with areas of hemorrhage and blackish discoloration.

Histologically $80-85 \%$ cases of MPNST consist of spindle cell population arranged in fascicles similar to fibrosarcoma. High grade tumors are hypercellular with increased nucleo-cytoplasmic ratio and 4 or more mitotic figures/hpf. Fifteen percent of the tumor may show variable differentiation with skeletal muscle, cartilage, bone, smooth muscle, glandular and lipomatous component. 8,9

$\mathrm{S}-100$ is considered to be the best marker for MPNST and shows positivity in $50-90 \%$ of cases. ${ }^{10}$ Nestin which is an intermediate filament protein is also sensitive for MPNST and can be used in combination with other markers in the diagnosis of MPNST. ${ }^{11}$ Another marker Leu -7 , is a known Schwann cell marker, is positive in approximately $50 \%$ cases of MPNST but it is less superior than S100. MPNST shows reactivity to different immuno markers according to their differentiation such as 
epithelioid MPNST of skin shows positivity to HMB-45 and MPNST with glandular differentiation is reactive to keratin, EMA and chromogranin. ${ }^{12}$ Findings on imaging studies define location and extention of the disease and reveals metastasis. Treatment is predominantly surgical and the goal of operation is to achieve complete surgical excision of the tumor with negative margins. This offers the best outcome with respect to both local recurrence and distant metastases. ${ }^{7,11}$ Radiation therapy can be employed in preoperative, intraoperative, and postoperative settings and treatment with adjuvant radiotherapy has yielded a statistically significant reduction in the rates of local disease recurrence. ${ }^{8}$ MPNST is locally invasive and a highly aggressive tumor with metastasis in $39 \%$ of cases and lungs being the most common secondary site. ${ }^{8}$ Local recurrence rate of MPNST has been reported to be $40-65 \%$ with 5 year survival of $16-52 \%{ }^{12}$ One study shows an overall survival rate of $84 \%$, which can be largely attributed to improved imaging leading to early diagnosis and aggressive treatment, employing adjuvant and neoadjuvant treatment modalities such as chemotherapy and radiation. ${ }^{12}$

\section{CONCLUSION}

MPNST can arise in any unusual site and the suspicion should arise in a rapidly growing painless tumor in and around the nerve tissue. Complete surgical removal should be the goal of treatment with definitive histological diagnosis.

\section{REFERENCES}

1. Panigrahi S, Mishra S, Das S, Dhir M. Primary malignant peripheral nerve sheath tumor at unusual location. Journal of Neurosciences in Rural Practice;available from: http://dx.doi.org/10.4103/0976-3147.116480

2. Aydin MD, Yildirim U, Gundogdu C, Dursun O, Uysal $\mathrm{HH}$, Ozdikici M. Malignant Peripheral Nerve Sheath Tumor of the Orbit: Case Report and Literature Review. Skull Base; 2004 May;14(2):109-13. Available from: http://dx.doi.org/10.1055/s-2004$\underline{828705}$

3. Patnaik A, Mishra S, Senapati S, Patra S, Tripathy K, Burma S. Primary intraosseous malignant peripheral nerve sheath tumor of spine with a giant paraspinal and retrospinal subcutaneous extension. Surgical Neurology International; 2012;3(1):157. Available from: http://dx.doi.org/10.4103/2152$\underline{7806.105096}$

4. Iwama $\mathrm{Y}$, Kunisada M, Goto H, Ohno Y, Yamashita J, Funasaka $Y$, et al. Malignant peripheral nerve sheath tumor of the distal phalanx of the fifth toe: a case report. Acta Radiologica Short Reports; 2014 Jan 15;3(1). Available from: http://dx.doi.org/10.1177/2047981613516033

5. Kar M, Deo SS, Shukla N, Malik A, DattaGupta S, Mohanti B, et al. World Journal of Surgical Oncology;4(1):55. Available from: http://dx.doi.org/10.1186/1477-7819-4-55

6. Cashen DV, Parisien RC, Raskin K, Hornicek FJ, Gebhardt MC, Mankin HJ. Survival Data for Patients with Malignant Schwannoma. Clinical Orthopaedics and Related Research;426:69-73. Availablefrom: http://dx.doi.org/10.1097/01.blo.0000131256.8245 $\underline{5 . c 5}$

7. Yu J, Deshmukh H, Payton JE, Dunham C, Scheithauer BW, Tihan T, et al. Array-Based Comparative Genomic Hybridization Identifies CDK4 and FOXM1 Alterations as Independent Predictors of Survival in Malignant Peripheral Nerve Sheath Tumor. Clinical Cancer Research; 2011 Feb 16;17(7):1924-34. Available from: http://dx.doi.org/10.1158/1078-0432.ccr-10-1551

8. Weiss SW. Enzinger and Weiss's Soft Tissue Tumors. Edited, $3^{\text {rd }}$ Ed, St. Louis, Mosby, Inc., 2001; pp 234-245.

9. Guo A, Liu A, Wei L, Song X. Malignant Peripheral Nerve Sheath Tumors: Differentiation Patterns and Immunohistochemical Features - A Mini-Review and Our New Findings. Journal of Cancer 2012;3:303-9. Available from: http://dx.doi.org/10.7150/jca.4179

10. Devadoss C, Rau A, Manjari S, Hasaf M. Paediatric malignant peripheral nerve sheath tumor with osteoid, rhabdomyosarcomatous, and liposarcomatous differentiation. Journal of Postgraduate Medicine2014;60(3):348. Available from: http://dx.doi.org/10.4103/0022-3859.138835

11.

LIVOLSI V. Rosai and Ackerman's surgical pathology, vols $1 \& 2$, 9th ed.J. Rosai. St Louis, MO, Mosby, 2004, 3136 pp + cd-rom, \$395.00. Human Pathology; 2004 Dec;35(12):1572. Available from: http://dx.doi.org/10.1016/s0046-8177(04)00455-1

12. Sheikh OA, Reaves A, Kralick FA, Brooks A, Musial RE, Gasperino J. Malignant Nerve Sheath Tumor of the Spinal Accessory Nerve: A Unique Presentation of a Rare Tumor. Journal of Clinical Neurology; 2012;8(1):75. Available from: http://dx.doi.org/10.3988/jcn.2012.8.1.75 